Comment. Math. Helv. 72 (1997) 266-284

(C) 1997 Birkhäuser Verlag, Basel

$0010-2571 / 97 / 020266-19 \$ 1.50+0.20 / 0$

Commentarii Mathematici Helvetici

\title{
Stable equivalence preserves representation type
}

\author{
Henning Krause
}

\begin{abstract}
Given two finite dimensional algebras $\Lambda$ and $\Gamma$, it is shown that $\Lambda$ is of wild representation type if and only if $\Gamma$ is of wild representation type provided that the stable categories of finite dimensional modules over $\Lambda$ and $\Gamma$ are equivalent. The proof uses generic modules. In fact, a stable equivalence induces a bijection between the isomorphism classes of generic modules over $\Lambda$ and $\Gamma$, and the result follows from certain additional properties of this bijection. In the second part of this paper the Auslander-Reiten translation is extended to an operation on the category of all modules. It is shown that various finiteness conditions are preserved by this operation. Moreover, the Auslander-Reiten translation induces a homeomorphism between the set of non-projective and the set of non-injective points in the Ziegler spectrum. As a consequence one obtains that for an algebra of tame representation type every generic module remains fixed under the Auslander-Reiten translation.
\end{abstract}

Mathematics Subject Classification (1991). 16G60, 16D90, 18E30.

Keywords. Stable equivalence, generic module, Ziegler spectrum, Auslander-Reiten translation.

Given a finite dimensional algebra $\Lambda$ over an algebraically closed field one frequently disregards the projective objects in the category mod $\Lambda$ of finite dimensional $\Lambda$-modules and focuses on the stable category $\underline{\bmod } \Lambda$. The objects of $\underline{\bmod } \Lambda$ are those of $\bmod \Lambda$ but the Hom-groups are the ordinary Hom-groups modulo the subgroup of those morphisms which factor through a projective $\Lambda$-module.

In this paper we show that $\underline{\bmod } \Lambda$ determines the representation type of $\Lambda$. Recall that the algebra $\Lambda$ is either tame, i.e. all finite dimensional indecomposable $\Lambda$-modules of a given dimension belong to finitely many one-parameter families, or $\Lambda$ is wild, i.e. there are non-trivial two-parameter families of finite dimensional indecomposable $\Lambda$-modules [9]. Of course, one feels that this dichotomy should not depend on the deletion of finitely many objects in the category mod $\Lambda$, and this is precisely one of the main results of this paper. More precisely, given another algebra $\Gamma$ and an equivalence $\underline{\bmod } \Lambda \rightarrow \underline{\bmod } \Gamma$, then $\Gamma$ is tame if $\Lambda$ is tame. Moreover, under such an equivalence the one-parameter families in $\bmod \Lambda$ correspond to the ones in $\bmod \Gamma$. The fact that $\underline{\bmod } \Lambda$ determines the representation type of $\Lambda$ also follows, for some classes of symmetric algebras, from recent work of Assem, de la Peña and Erdmann [2, 10]; however their methods are completely different.

Equivalences between stable module categories have been studied by many au- 
thors. They naturally occur for instance in representation theory of finite groups. Another source of examples, which includes every algebra of Loewy length 2, is the class of algebras stably equivalent to a hereditary algebra. Usually the analysis concentrates on homological properties of the category mod $\Lambda$ which are preserved by an equivalence $\underline{\bmod } \Lambda \rightarrow \underline{\bmod } \Gamma$. In this paper we follow a different approach. We investigate pure-injective modules which are not necessarily finitely presented. Among them the endofinite modules are of particular interest. Recall that a module is endofinite if it is of finite length when regarded in the natural way as a module over its endomorphism ring. In order to study the non-finitely presented $\Lambda$-modules we introduce a new category $\underline{\lim } \underline{\bmod } \Lambda$ which is essentially determined by the following three properties:

(i) $\underline{\lim } \underline{\bmod } \Lambda$ contains, up to equivalence, $\underline{\bmod } \Lambda$ as a full subcategory.

(ii) $\underline{\lim } \underline{\bmod } \Lambda$ is an additive category with direct limits.

(iii) Every object in $\underline{\lim } \underline{\bmod } \Lambda$ is a direct limit of objects in $\underline{\bmod } \Lambda$.

The canonical functor $\overrightarrow{\bmod } \Lambda \rightarrow \underline{\bmod } \Lambda$ induces a functor $\operatorname{Mod} \Lambda \rightarrow \underline{\lim } \underline{\bmod } \Lambda$, and we use it to establish a close relationship between the endofinite objects in $\operatorname{Mod} \Lambda$ and $\underline{\underline{\bmod } \Lambda} \Lambda$. By Crawley-Boevey's characterization of tameness in terms of endofinite indecomposables [6], we can then deduce that the representation type of $\Lambda$ is determined by $\underline{\lim } \underline{\bmod } \Lambda$, and hence by $\underline{\bmod } \Lambda$.

The second part of this paper is devoted to the Auslander-Reiten translation $M \mapsto D \operatorname{Tr} M$ which Auslander and Reiten introduced in [4] for finitely presented modules over an artin algebra $\Lambda$, and which plays a prominent role in modern representation theory. We extend this to an operation on the category of all $\Lambda$-modules and show that most of the basic properties for finitely presented modules carry over to arbitrary modules. Moreover, finiteness conditions like pure-injectivity and endofiniteness are preserved. Finally we show that the Auslander-Reiten translation induces a homeomorphism between the set of non-projective points and the set of non-injective points in the Ziegler spectrum of $\Lambda$. As a consequence we obtain that for a tame algebra every generic module remains fixed under the Auslander-Reiten translation. Recall that a module is generic if it is indecomposable endofinite but not finitely presented. Therefore our result complements Crawley-Boevey's theorem which states for a tame algebra that for every natural $n$ almost all finitely presented indecomposable modules of endolength $n$ are fixed under the Auslander-Reiten translation [5].

The final section of this paper is devoted to a remarkable property of certain triangulated categories which follows from our discussion of stable categories. We show that for every quasi-Frobenius ring $\Lambda$ of finite representation type the triangulation on $\underline{\bmod } \Lambda$ induces a triangulation on $\underline{\lim \bmod } \Lambda$. We obtain therefore non-trivial examples of triangulated categories having the property that the triangulation induces a triangulation on the category of ind-objects in the sense of Grothendieck and Verdier [12]. 


\section{Locally finitely presented categories}

We recall some terminology and some well-known facts about locally finitely presented categories. Most of this material can be found in Crawley-Boevey's exposition [8]. Recall that a direct limit in a category $\mathcal{A}$ is the colimit of a functor $I \rightarrow \mathcal{A}$ where $I$ is a directed set. Now let $\mathcal{A}$ be an additive category in which direct limits exist. An object $X$ in $\mathcal{A}$ is finitely presented provided that the representable functor $\operatorname{Hom}(X$,$) commutes with direct limits and we denote by \operatorname{fp}(\mathcal{A})$ the full subcategory of finitely presented objects in $\mathcal{A}$. The category $\mathcal{A}$ is said to be locally finitely presented if the isomorphism classes of $\operatorname{fp}(\mathcal{A})$ form a set and every object in $\mathcal{A}$ is a direct limit of objects in $\operatorname{fp}(\mathcal{A})$.

Suppose now that $\mathcal{C}$ is a skeletally small additive category. We shall construct a locally finitely presented category $\underline{\lim \mathcal{C}}$ which is essentially determined by the following three properties:

(i) $\underline{\lim } \mathcal{C}$ contains, up to equivalence, $\mathcal{C}$ as a full subcategory.

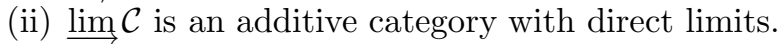

(iii) Every object in $\underline{\lim } \mathcal{C}$ is a direct limit of objects in $\mathcal{C}$.

Denote by $\left(\mathcal{C}^{\mathrm{op}}, \mathrm{Ab}\right)$ the category of additive functors $\mathcal{C}^{\mathrm{op}} \rightarrow \mathrm{Ab}$ into the category of abelian groups. As for module categories, there is a tensor product $\left(\mathcal{C}^{\mathrm{op}}, \mathrm{Ab}\right) \times$ $(\mathcal{C}, \mathrm{Ab}) \rightarrow \mathrm{Ab},(X, Y) \mapsto X \otimes_{\mathcal{C}} Y$, and a functor $X$ in $\left(\mathcal{C}^{\mathrm{op}}, \mathrm{Ab}\right)$ is flat if $X \otimes_{\mathcal{C}}-$ is exact. The full subcategory of flat functors is denoted by $\operatorname{Flat}\left(\mathcal{C}^{\mathrm{op}}, \mathrm{Ab}\right)$; it is locally finitely presented and the finitely presented objects in $\operatorname{Flat}\left(\mathcal{C}^{\mathrm{op}}, \mathrm{Ab}\right)$ are precisely the direct summands of representable functors. Moreover given any locally finitely presented category $\mathcal{A}$, the functor

$$
\mathcal{A} \longrightarrow \operatorname{Flat}\left(\mathrm{fp}(\mathcal{A})^{\mathrm{op}}, \mathrm{Ab}\right),\left.\quad X \mapsto \operatorname{Hom}(, X)\right|_{\mathrm{fp}(\mathcal{A})}
$$

is an equivalence [8, Theorem 1.4]. We now define $\underline{\lim } \mathcal{C}=\operatorname{Flat}\left(\mathcal{C}^{\text {op }}, \mathrm{Ab}\right)$ and it follows from our discussion that $\lim \mathcal{C}$ satisfies the conditions (i) - (iii). The following property of $\underline{\lim \mathcal{C}}$ well be needed later.

Lemma 1.1. Any additive functor $\mathcal{C} \rightarrow \mathcal{A}$ into an additive category with direct limits extends, up to isomorphism, uniquely to a functor $\underline{\lim } \mathcal{C} \rightarrow \mathcal{A}$ which commutes with direct limits.

Proof. See [17, Property 5.6].

Given a locally finitely presented category $\mathcal{A}$ and a full additive subcategory $\mathcal{C}$ of $\operatorname{fp}(\mathcal{A})$, then the inclusion $\mathcal{C} \rightarrow \mathcal{A}$ induces a fully faithful functor $\lim _{\longrightarrow} \mathcal{C} \mathcal{A}$ which identifies $\lim \mathcal{C}$ with the full subcategory of $\mathcal{A}$ formed by the direct limits of objects in $\mathcal{C}$. In the sequel we shall therefore view $\underline{\lim } \mathcal{C}$ as a full subcategory of $\mathcal{A}$.

Suppose now that $\mathcal{A}$ is a locally finitely presented category which has products. There is a notion of purity for $\mathcal{A}$ which is defined as follows. A sequence of 
morphisms $0 \rightarrow L \stackrel{\varphi}{\rightarrow} M \stackrel{\psi}{\rightarrow} N \rightarrow 0$ in $\mathcal{A}$ is pure-exact if the induced sequence $0 \rightarrow \operatorname{Hom}(X, L) \rightarrow \operatorname{Hom}(X, M) \rightarrow \operatorname{Hom}(X, N) \rightarrow 0$ is exact for every $X$ in $\operatorname{fp}(\mathcal{A})$. In this case the morphism $\varphi$ is called a pure monomorphism, and $L$ is pureinjective if every pure monomorphism $L \rightarrow M$ splits. We denote by $\operatorname{Pinj} \mathcal{A}$ the full subcategory of pure-injective objects. One can assign to $\mathcal{A}$ a locally coherent Grothendieck category $D(\mathcal{A})$ and a fully faithful functor $d: \mathcal{A} \rightarrow D(\mathcal{A})$ which identifies the pure-exact sequences $0 \rightarrow L \rightarrow M \rightarrow N \rightarrow 0$ in $\mathcal{A}$ with the exact sequences $0 \rightarrow d(L) \rightarrow d(M) \rightarrow d(N) \rightarrow 0$ in $D(\mathcal{A})$. Moreover $d$ identifies the pure-injective objects in $\mathcal{A}$ with the injective objects in $D(\mathcal{A})$ [8, Theorem 3.3].

A full subcategory of $\mathcal{A}$ is called definable if it is closed under direct limits, products and pure subobjects taken in $\mathcal{A}$. Finally we recall that Zsp $\mathcal{A}$ denotes the Ziegler spectrum of $\mathcal{A}$ which is a topological space. The points are the isomorphism classes of indecomposable pure-injective objects in $\mathcal{A}$ and the assignment $\mathcal{X} \mapsto$ $\mathcal{X} \cap Z \operatorname{sp} \mathcal{A}$ defines a bijection between the definable subcategories of $\mathcal{A}$ and the closed subsets of Zsp $\mathcal{A}$. This follows from Corollary 4.7 and Theorem 6.2 in [18]. If $\mathcal{A}=\operatorname{Mod} \Lambda$ is a module category, then we simplify our notation and write $\operatorname{Pinj} \Lambda, Z \operatorname{zsp} \Lambda$ etc.. Note that $Z \operatorname{sp} \Lambda$ is a quasi-compact space [24], see also [16, Proposition 4.7].

\section{Stable module categories}

Let $\Lambda$ be a ring. The category $\operatorname{Mod} \Lambda$ of (right) $\Lambda$-modules is locally finitely presented and we denote by $\bmod \Lambda$ the full subcategory of finitely presented $\Lambda$ modules. In this section we define the stable module category $\underline{\bmod }_{\mathcal{C}} \Lambda$ with respect to a full additive subcategory $\mathcal{C}$ of $\bmod \Lambda$ and discuss the basic properties of the corresponding locally finitely presented category $\underline{\lim }_{\underline{\bmod }} \Lambda$. We begin with the following definition.

Let $\mathcal{A}$ be an additive category and suppose that $\mathcal{C}$ is an additive subcategory of $\mathcal{A}$. Then one can form the stable quotient category $\mathcal{A}\lceil\mathcal{C}$ which is additive and admits an additive quotient functor $q: \mathcal{A} \rightarrow \mathcal{A}\lceil\mathcal{C}$ such that $q(\mathcal{C})=0$. The objects of $\mathcal{A}\lceil\mathcal{C}$ are those of $\mathcal{A}$ and for every pair $X, Y$ of objects $\operatorname{Hom}(X, Y)$ is the group of morphisms in $\mathcal{A}$ modulo those which factor through an object in $\mathcal{C}$. The quotient functor $q$ is characterized by the property that every additive functor $f: \mathcal{A} \rightarrow \mathcal{B}$ between additive categories with $f(\mathcal{C})=0$ induces a unique additive functor $\bar{f}: \mathcal{A}\left\lceil\mathcal{C} \rightarrow \mathcal{B}\right.$ such that $f=\bar{f}_{\circ} q$. If $\mathcal{C}$ is an additive subcategory of $\bmod \Lambda$, then we denote $\bmod \Lambda\left\lceil\mathcal{C}\right.$ by $\underline{\bmod }_{\mathcal{C}} \Lambda$.

Proposition 2.1. Let $\mathcal{C}$ be a full additive subcategory of $\bmod \Lambda$. Then there exists an additive category with direct limits $\varliminf^{\operatorname{limod}} \underline{\bmod }_{\mathcal{C}} \Lambda$ and a functor $q: \operatorname{Mod} \Lambda \rightarrow$ $\varliminf_{(1)} \underline{\bmod }_{\mathcal{C}} \Lambda$ having the following properties.

(1) $q$ commutes with direct limits and $q(M)=0$ if and only if $M$ is a direct limit of modules in $\mathcal{C}$. 
(2) Let $f: \operatorname{Mod} \Lambda \rightarrow \mathcal{A}$ be a functor into an additive category with direct limits. If $f$ commutes with direct limits and $f(M)=0$ for all $M$ in $\mathcal{C}$, then there is, up to isomorphism, a unique functor $\bar{f}: \varliminf^{\bmod } \underline{\bmod }_{\mathcal{C}} \Lambda \rightarrow \mathcal{A}$ which commutes with direct limits and satisfies $f=\bar{f} \circ q$.

(3) $\varliminf_{\bmod } \underline{\bmod }_{\mathcal{C}} \Lambda$ is locally finitely presented and $q$ induces an equivalence between $\underline{\bmod }_{\mathcal{C}} \Lambda$ and the full subcategory of finitely presented objects in $\underline{\lim }_{\underline{\bmod }} \Lambda$.

Proof. The category $\underline{\lim }_{\underline{\bmod }} \underline{\mathcal{C}}_{\mathcal{C}} \Lambda$ has been defined in the preceding section and it follows from Lemma 1.1 that the quotient functor $\bmod \Lambda \rightarrow \underline{\bmod }_{\mathcal{C}} \Lambda$ induces, up to isomorphism, a unique functor $q: \operatorname{Mod} \Lambda \rightarrow \underline{\lim }_{\bmod } \mathcal{C} \Lambda$ which commutes with direct limits.

(1) Clearly $q(M)=0$ for every $M \in \underline{\lim } \mathcal{C}$ since $q$ commutes with direct limits. Thus we need to show that $q(M)=0$ implies $M \in \underline{\lim \mathcal{C}}$. By [8, Lemma 4.1] it is sufficent to show that every morphism $\varphi: X \rightarrow M$ with $X \in \bmod \Lambda$ factors through an object in $\mathcal{C}$. To this end write $M=\underline{\lim } M_{i}$ as direct limit of finitely presented modules with canonical morphisms $\mu_{i}: M_{i} \rightarrow M$. If $K_{i}$ is the kernel of the morphisms $\operatorname{Hom}\left(X, M_{i}\right) \rightarrow \operatorname{Hom}\left(q(X), q\left(M_{i}\right)\right)$, then since the direct limit sequence $0 \rightarrow \underline{\lim } K_{i} \rightarrow \operatorname{Hom}(X, M) \rightarrow \operatorname{Hom}(q(X), q(M))$ is exact, $\varphi$ arises from some $\alpha \in K_{i}$. Thus $\varphi=\mu_{i} \circ \alpha$ and $\alpha$ factors through some object in $\mathcal{C}$. We conclude that $M \in \varliminf$ $\lim _{\text {. }}$

(2) The restriction $g=\left.f\right|_{\bmod \Lambda}$ induces a unique functor $\bar{g}: \underline{\bmod }_{\mathcal{C}} \Lambda \rightarrow \mathcal{A}$ such that $g(X)=\bar{g} \circ q(X)$ for every $X \in \bmod \Lambda$. The functor $\bar{g}$ extends by Lemma 1.1 uniquely to a functor $\bar{f}: \underline{\lim }_{\bmod _{\mathcal{C}}} \Lambda \rightarrow \mathcal{A}$ which commutes with direct limits.

(3) Clear from the construction.

For the rest of this paper we shall work with an additional finiteness condition on the subcategory $\mathcal{C}$ of $\bmod \Lambda$. Recall that $\mathcal{C}$ is a covariantly finite subcategory provided that every object $X \in \bmod \Lambda$ has a left $\mathcal{C}$-approximation, i.e. a morphism $X \rightarrow Y$ with $Y \in \mathcal{C}$ such that the induced map $\operatorname{Hom}\left(Y, Y^{\prime}\right) \rightarrow \operatorname{Hom}\left(X, Y^{\prime}\right)$ is surjective for every $Y^{\prime} \in \mathcal{C}$.

Proposition 2.2. Let $\mathcal{C}$ be a full additive subcategory of $\bmod \Lambda$ and suppose that

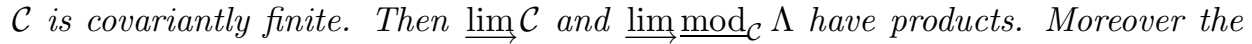
canonical functors $i: \varliminf_{\longrightarrow} \mathcal{C} \rightarrow \underline{\operatorname{Mod} \Lambda}$ and $q: \operatorname{Mod} \Lambda \rightarrow \varliminf_{\operatorname{limod}} \Lambda$ have the following properties.

(1) $i$ and $q$ commute with products.

(2) $i$ and $q$ send pure-exact sequences to pure-exact sequences and pure-injective objects to pure-injective objects.

(3) Let $\mathcal{X}=\operatorname{Pinj} \Lambda \cap \lim _{\mathcal{C}}$. Then $q$ induces an equivalence between $\operatorname{Pinj} \Lambda\lceil\mathcal{X}$ and the full subcategory of pure-injective objects in $\underline{\lim }_{\underline{\bmod }} \Lambda$.

(4) Zsp $\Lambda \cap \underline{\lim } \mathcal{C}$ is a closed subset of Zsp $\Lambda$ and $q$ induces a homeomorphism

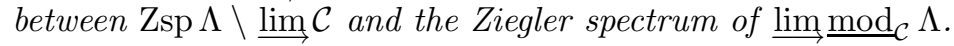

(5) Let $M \in \operatorname{Zsp} \Lambda \backslash \underline{\lim } \mathcal{C}$ and $N=q(M)$. Then $q$ induces an isomorphism 
between $\operatorname{End}_{\Lambda}(M) / \operatorname{rad}_{\operatorname{End}}(M)$ and $\operatorname{End}(N) / \operatorname{rad} \operatorname{End}(N)$.

Proof. It follows from [18, Proposition 4.13] that $\mathfrak{\operatorname { l i m }}_{\mathrm{C}}$ is a definable subcategory

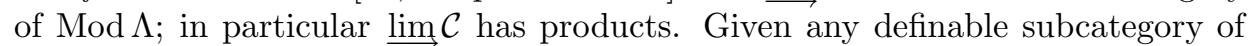
$\operatorname{Mod} \Lambda$ there is a corresponding definable quotient category in the sense of [18]. Such a category has automatically products and in our situation the definable quotient category corresponding to $\underline{\lim }_{\mathrm{C}}$ is exactly $\underline{\lim }_{\underline{\bmod }} \Lambda$; this follows from [18, Theorem 5.4]. The canonical functors $i$ and $q$ are definable in the sense of [18] and we refer to Theorem 7.2, Theorem 7.8, Theorem 5.1 and Corollary 6.3 in [18] for the proofs of (1) - (5).

Given a full additive subcategory $\mathcal{C}$ of $\bmod \Lambda$, then it follows from Proposition 2.1 that the canonical functor $\operatorname{Mod} \Lambda \rightarrow \underline{\lim }_{\bmod _{\mathcal{C}}} \Lambda$ induces a functor $\operatorname{Mod} \Lambda\left\lceil\underline{\lim } \mathcal{C} \rightarrow \underline{\lim }_{\bmod _{\mathcal{C}}} \Lambda\right.$. The next result shows that this functor is an equivalence if $\Lambda$ is of finite representation type.

Corollary 2.3. Let $\Lambda$ be a ring of finite representation type and suppose that $\mathcal{C}$ is a full additive subcategory of $\bmod \Lambda$. Then the canonical functor $\operatorname{Mod} \Lambda \rightarrow$ $\varliminf_{\underline{\bmod }} \Lambda$ induces an equivalence between $\operatorname{Mod} \Lambda\left\lceil\varliminf_{\mathcal{l}} \mathcal{C}\right.$ and $\varliminf_{\bmod } \Lambda$. In particular the category $\operatorname{Mod} \Lambda\rfloor \overleftrightarrow{\mathrm{lim} \mathcal{C}}$ is locally finitely presented and has products.

Proof. If $\Lambda$ is of finite representation type, then any additive subcategory of $\bmod \Lambda$ is covariantly finite. Moreover, any $\Lambda$-module is pure-injective since it has the descending chain condition on subgroups of finite definition $[3,13]$. Therefore also every object in $\varliminf_{\mathfrak{m o d}} \underline{\bmod }_{\mathcal{C}} \Lambda$ is pure-injective. The assertion now follows from part (3) of the preceding proposition.

\section{Endofinite modules}

Recall that a $\Lambda$-module $M$ is endofinite if it is of finite length when regarded in the natural way as an $\operatorname{End}_{\Lambda}(M)^{\mathrm{op}}$-module. This definition can be generalized as follows. Let $M$ be an object of a locally finitely presented category $\mathcal{A}$ with products. Then $M$ is endofinite if $\operatorname{Hom}(X, M)$ is of finite length as an $\operatorname{End}(M)^{\mathrm{op}}$-module for all $X \in \operatorname{fp}(\mathcal{A})$. Furthermore $M$ is generic if $M$ is indecomposable endofinite but not finitely presented. Note that every endofinite object $M$ is $\Sigma$-pure-injective, i.e. every coproduct of copies of $M$ is pure-injective [8, Theorem 3.5.2]. We shall use the following characterization.

Lemma 3.1. For any object $M$ in $\mathcal{A}$ the following are equivalent.

(1) $M$ is endofinite.

(2) There is a closed subset $\mathcal{U}$ of $\mathrm{Zsp} \mathcal{A}$ satisfying 
(i) $\mathcal{U}$ carries the discrete topology.

(ii) Every product of copies of $M$ is a coproduct of copies of objects in $\mathcal{U}$.

(3) Any pure-injective object which belongs to the smallest definable subcategory of $\mathcal{A}$ containing $M$, is endofinite.

Proof. The part $(1) \Leftrightarrow(2)$ is [18, Corollary 12.13] and (1) $\Leftrightarrow(3)$ follows from [18, Theorem 10.2] since the endocategory of any object belonging to the definable subcategory generated by $M$ is a quotient of the endocategory of $M$.

Our aim is now to discuss the relation between the endofinite $\Lambda$-modules and the endofinite objects of $\underline{\lim }_{\bmod } \Lambda$. To this end another definition is needed. Given a finitely presented indecomposable endofinite $\Lambda$-module $M$ with $\Gamma=\operatorname{End}_{\Lambda}(M)^{\text {op }}$ we define $D M=\operatorname{Hom}_{\Gamma}(M, I)$ where $I$ is the injective envelope of $\Gamma / \operatorname{rad} \Gamma$. Note that $D M$ is automatically an indecomposable endofinite $\Lambda^{\mathrm{op}}$-module. The ring $\Lambda$ is called right dualizing if $D M$ is also finitely presented for every such $M$, and $\Lambda$ is dualizing if both $\Lambda$ and $\Lambda^{\mathrm{op}}$ are right dualizing. Important examples of dualizing rings are noetherian algebras [7] and artinian PI-rings [21]. Recall that $\Lambda$ is a noetherian algebra if the centre $Z(\Lambda)$ is noetherian and $\Lambda$ is a finitely generated $Z(\Lambda)$-module. Given any object $X$ we denote by add $X$ the full subcategory of all finite coproducts of direct summands of $X$.

Proposition 3.2. Let $X$ be a finitely presented endofinite $\Lambda$-module and choose a complete set $\left\{X_{1}, \ldots, X_{n}\right\}$ of pairwise non-isomorphic indecomposable direct summands of $X$. Let $\mathcal{C}=\operatorname{add} X$. Then $\mathcal{C}$ is a covariantly finite subcategory of $\bmod \Lambda$ and $\underline{\lim C}$ is the full subcategory of coproducts of copies of the $X_{i}$ 's. Now suppose that $\vec{D} X_{i}$ is a finitely presented $\Lambda^{\mathrm{op}}$-module for all $i$. Then any $\Lambda$-module $M$ is endofinite if and only if the canonical functor $\operatorname{Mod} \Lambda \rightarrow \underline{\lim }_{\underline{\bmod }} \Lambda$ sends $M$ to an endofinite object.

Proof. To show that $\mathcal{C}$ is covariantly finite let $Y$ be any finitely presented $\Lambda$-module. The $\operatorname{End}_{\Lambda}(X)^{\mathrm{op}}$-module $\operatorname{Hom}_{\Lambda}(Y, X)$ is of finite length and has therefore a set of generators $\varphi_{1}, \ldots, \varphi_{r}$. It is clear that the corresponding morphism $\left(\varphi_{i}\right): Y \rightarrow$ $\coprod_{i=1}^{r} X$ is a left $\mathcal{C}$-approximation. The module $X$ is $\Sigma$-pure-injective and therefore $\lim _{\mathcal{C}}$ coincides with the definable subcategory generated by $X$. More precisely, the coproducts of copies of the $X_{i}$ 's form a definable subcategory by [19, Corollary 9.8] which is therefore the smallest subcategory containing $\mathcal{C}$ and all direct limits of modules in $\mathcal{C}$.

To prove the rest of the assertion consider the sequence of functors

$$
D\left(\underline{\lim }_{\underline{\bmod }_{\mathcal{C}}} \Lambda\right) \stackrel{q^{*}}{\longrightarrow} D(\Lambda) \stackrel{i^{*}}{\longrightarrow} D(\underline{\lim } \mathcal{C})
$$

as in the proof of [18, Theorem 5.1]. We shall identify any locally finitely presented category $\mathcal{A}$ with the image of the fully faithful functor $d: \mathcal{A} \rightarrow D(\mathcal{A})$ mentioned in Section 1. Let us recall from [18] some properties of $q^{*}$ and $i^{*}$. The functor $q^{*}$ 
identifies $D\left(\underline{\lim }_{\underline{\bmod }} \Lambda\right)$ with a localizing subcategory $\mathcal{T}$ of $D(\Lambda)$ and $i^{*}$ induces an equivalence between $D(\Lambda) / \mathcal{T}$ and $D\left(\varliminf_{\lim } \mathcal{C}\right)$. The functor $q^{*}$ has a right adjoint which coincides with $q: \operatorname{Mod} \Lambda \rightarrow \underline{\lim } \underline{\bmod }_{\mathcal{C}} \Lambda$ when restricted to Mod $\Lambda$; similarly $i^{*}$ has a right adjoint which coincides with $i: \lim _{\vec{C}} \rightarrow \operatorname{Mod} \Lambda$ when restricted to $\underline{\lim \mathcal{C}}$. We have already seen that Zsp $\lim _{\mathfrak{C}}=\left\{\vec{X}_{1}, \ldots, X_{n}\right\}$ and we observe that this is an open subset of Zsp $\Lambda$. In fact our assumption on $X$ implies that $\left\{X_{i}\right\}$ is open for every $i$ by [19, Lemma 4.4]. Suppose now that $M$ is any pure-injective $\Lambda$-module and let $N=q(M)$. We shall use the fact that there is a decomposition $M=E(N) \amalg M^{\prime}$ where $E(N)$ denotes the injective envelope in $D(\Lambda)$ and $M^{\prime}$ belongs to $\varliminf_{\mathcal{C}} \mathcal{C}$ [11, III, Corollaire 3.2].

Suppose first that $M$ is endofinite and let $\mathcal{U}$ be the corresponding subset of Zsp $\Lambda$ as in Lemma 3.1. Taking the set $\mathcal{V}=\{q(L) \mid L \in \mathcal{U}, q(L) \neq 0\}$ one shows immediately that $N$ is endofinite. Condition (i) in Lemma 3.1 follows from Proposition 2.2 and (ii) is clear since $q$ commutes with products and coproducts.

Now suppose that $N$ is endofinite and let $\mathcal{V}$ be the corresponding subset as in Lemma 3.1. We claim that $\mathcal{U}=\{L \in \operatorname{Zsp} \Lambda \mid q(L) \in \mathcal{V}\} \cup\left\{X_{1}, \ldots, X_{n}\right\}$ is a subset of Zsp $\Lambda$ which satisfies (i) - (ii) in Lemma 3.1. Observe first that for every $L$ in $\mathcal{V}$ the injective envelope $E(L)$ lies in $\mathcal{U}$ since $q(E(L))=L$. It follows from Proposition 2.2 that (i) holds since $\left\{X_{i}\right\}$ is open for every $i$ and (i) holds for $\mathcal{V}$. Now consider a product $\prod_{I} M$. We find a decomposition $\prod_{I} N=\coprod_{j \in J} N_{j}$ with $N_{j} \in \mathcal{V}$ for all $j$ and therefore $\prod_{I} M=\left(\coprod_{j \in J} E\left(N_{j}\right)\right) \amalg M^{\prime}$ with $M^{\prime} \in \varliminf_{\longrightarrow} \mathcal{C}$ since taking injective envelopes commutes with arbitrary coproducts. We have already shown that $M^{\prime}$ is a coproduct of copies of the $X_{i}^{\prime}$ 's and therefore (ii) holds. Thus $M$ is endofinite by Lemma 3.1 .

It remains to consider the case that $M$ is an arbitrary $\Lambda$-module and $N=q(M)$ is endofinite. We use now the characterization of endofiniteness in part (3) of Lemma 3.1. Replacing $M$ by any pure-injective $\Lambda$-module $M^{\prime}$ belonging to the smallest definable subcategory containing $M$ we deduce from the first part of the proof that $M^{\prime}$ is endofinite since $N^{\prime}=q\left(M^{\prime}\right)$ belongs to the smallest definable subcategory containing $N$. Thus $M$ is endofinite by Lemma 3.1 and the proof is complete.

Corollary3.3. Let $\Lambda$ be a right dualizing ring and suppose that $\mathcal{C}$ is a full additive subcategory of $\bmod \Lambda$ which is closed under direct summands. Then the following are equivalent.

(1) $\mathcal{C}=\operatorname{add} X$ for some endofinite $\Lambda$-module $X$.

(2) $\varliminf_{\bmod } \Lambda$ has products and any pure-injective $\Lambda$-module $M$ is endofinite if and only if the canonical functor $\operatorname{Mod} \Lambda \rightarrow \underline{\lim }_{\underline{\bmod }} \Lambda$ sends $M$ to an endofinite object.

Proof. One direction follows directly from the preceding proposition. Therefore suppose (2). Let $M=\coprod_{i \in I} X_{i}$ be the coproduct of a representative set of modules 
in $\mathcal{C}$. Clearly $\operatorname{Mod} \Lambda \rightarrow \underline{\lim }_{\bmod _{\mathcal{C}}} \Lambda$ sends $M$ to an endofinite object. Using Lemma 3.1 and the compactness of Zsp $\Lambda$ we find a set $\left\{M_{1}, \ldots, M_{n}\right\}$ in Zsp $\Lambda$ such that every object in $\mathcal{C}$ is a coproduct of copies of the $M_{i}$ 's. If $\left\{M_{1}, \ldots, M_{n}\right\}$ is minimal with respect to this property, then $\mathcal{C}=\operatorname{add} X$ for $X=\coprod_{i=1}^{n} M_{i}$ and therefore (1) is shown.

We continue with two further applications of Proposition 3.2. Following CrawleyBoevey [7] a ring $\Lambda$ is generically wild if there is a generic $\Lambda$-module $M$ such that $\operatorname{End}_{\Lambda}(M)$ is not a PI-ring. Note that for any endofinite $\Lambda$-module the endomorphism ring $\Gamma$ is a PI-ring iff $\Gamma / \operatorname{rad} \Gamma$ is a PI-ring since $\operatorname{rad} \Gamma$ is nilpotent. A finite dimensional algebra over an algebraically closed field is generically wild if and only it is wild in the usual sense [6, Theorem 4.4].

Corollary 3.4. Let $\Lambda$ and $\Gamma$ be dualizing rings. Suppose that $X$ is a finitely presented endofinite $\Lambda$-module and that $Y$ is a finitely presented endofinite $\Gamma$-module. Let $\mathcal{C}=\operatorname{add} X$ and $\mathcal{D}=\operatorname{add} Y$. Then any equivalence $f: \underline{\bmod }_{\mathcal{C}} \Lambda \rightarrow \underline{\bmod }_{\mathcal{D}} \Gamma$ induces a bijection $M \mapsto M_{f}$ between the isomorphism classes of generic modules in $\operatorname{Mod} \Lambda$ and $\operatorname{Mod} \Gamma$ such that $\operatorname{End}_{\Lambda}(M) / \operatorname{rad} \operatorname{End}_{\Lambda}(M) \operatorname{and}_{\operatorname{End}}\left(M_{f}\right) / \operatorname{rad}_{\operatorname{End}_{\Gamma}}\left(M_{f}\right)$ are isomorphic; in particular $\Lambda$ is generically wild if and only if $\Gamma$ is generically wild.

Proof. Given a dualizing ring it has been shown in [19, Theorem 4.7] that an indecomposable endofinite module $M$ is generic iff $\{M\}$ is not open in the Ziegler spectrum. Using this characterization the assertion follows from Proposition 2.2 and Proposition 3.2.

Suppose now that $\Lambda$ is an artin algebra. Given any generic $\Lambda$-module $M$, denote by $\operatorname{ind}_{M} \Lambda$ the isomorphism classes of finitely presented indecomposable $\Lambda$-modules which belong to a homogeneous tube $\mathcal{T}$ of the Auslander-Reiten quiver such that $M$ is the unique generic module belonging to the smallest definable subcategory of $\operatorname{Mod} \Lambda$ containing all modules in $\mathcal{T}$. Note that for any natural $n$ almost all indecomposable $\Lambda$-modules of endolength $n$ belong to $\bigcup_{M} \operatorname{ind}_{M} \Lambda$ provided that $\Lambda$ is a finite dimensional algebra over an algebraically closed field of tame representation type [19, Corollary 9.6]. We are now in a position to show that an equivalence $\underline{\bmod } \Lambda \rightarrow \underline{\bmod } \Gamma$ sends a family $\operatorname{ind}_{M} \Lambda$ to a family $\operatorname{ind}_{N} \Gamma$. As usual, $\underline{\bmod } \Lambda$ is the short notation for $\underline{\bmod }_{\operatorname{proj} \Lambda} \Lambda$ where $\operatorname{proj} \Lambda$ denotes the category of finitely presented projective $\Lambda$-modules.

Corollary 3.5. Let $\Lambda$ and $\Gamma$ be artin algebras and suppose that $f: \underline{\bmod } \Lambda \rightarrow \underline{\bmod } \Gamma$ is an equivalence. Then $f$ induces a bijection $M \mapsto M_{f}$ between the isomorphism classes of generic modules in $\operatorname{Mod} \Lambda$ and $\operatorname{Mod} \Gamma$ such that $f\left(\operatorname{ind}_{M} \Lambda\right)=\operatorname{ind}_{M_{f}} \Gamma$.

Proof. The equivalence $\underline{\bmod } \Lambda \rightarrow \underline{\bmod } \Gamma$ induces a bijection between the isomor- 
phism classes of indecomposable non-projective modules in $\bmod \Lambda$ and $\bmod \Gamma$. This bijection preserves homogeneous tubes and extends to a homeomorphism on the level of the Ziegler spectra by Proposition 2.2. We have seen in the preceding corollary that this homeomorphism induces a bijection $M \mapsto M_{f}$ between the generic modules in Zsp $\Lambda$ and Zsp $\Gamma$, and we conclude that $f\left(\operatorname{ind}_{M} \Lambda\right)=\operatorname{ind}_{M_{f}} \Gamma$ for every generic $M$, since a $\Lambda$-module lying in a homogeneous tube $\mathcal{T}$ belongs to ind $_{M} \Lambda$ if and only if $M$ is the unique generic module in the closure of $\mathcal{T}$ viewed as a subset of Zsp $\Lambda$.

\section{A generalization}

Our results from the preceding sections can be generalized. In fact we could replace a module category with any locally finitely presented category $\mathcal{A}$ which has products. We shall use the following properties:

(A1) If $M \in Z \operatorname{sp} \mathcal{A}$ is finitely presented and endofinite, then $\{M\}$ is open.

(A2) Every object in $\operatorname{fp}(\mathcal{A})$ is pure-injective.

The following result is formulated in this slightly more general context.

Theorem 4.1. Let $X$ be a finitely presented endofinite object in $\mathcal{A}$ and choose a complete set $\left\{X_{1}, \ldots, X_{n}\right\}$ of pairwise non-isomorphic indecomposable direct summands of $X$. Let $\mathcal{C}=\operatorname{add} X$ and $\mathcal{B}=\underline{\lim } \mathrm{fp}(\mathcal{A})\lceil\mathcal{C}$. Then the canonical functor $q: \mathcal{A} \rightarrow \mathcal{B}$ has the following properties.

(1) $q$ induces a homeomorphism $\operatorname{Zsp} \mathcal{A} \backslash\left\{X_{1}, \ldots, X_{n}\right\} \rightarrow \operatorname{Zsp} \mathcal{B}, M \mapsto M^{\prime}$, such that $\operatorname{End}(M) / \operatorname{rad} \operatorname{End}(M)$ and $\operatorname{End}\left(M^{\prime}\right) / \operatorname{rad} \operatorname{End}\left(M^{\prime}\right)$ are isomorphic.

(2) Suppose (A1). Then any object $M$ in $\mathcal{A}$ is endofinite if and only if $q(M)$ is endofinite.

(3) Suppose (A1) - (A2). Then q induces a bijection between the generic objects in $\mathcal{A}$ and $\mathcal{B}$.

Proof. Adapt the proof of Proposition 2.2 and Proposition 3.2. Observe that (3) is a consequence of (3) in Proposition 2.2. To see this let $M \in \mathrm{Zsp} \Lambda \backslash\left\{X_{1}, \ldots, X_{n}\right\}$ and $N=q(M)$. If $M$ is finitely presented, then $N$ is also finitely presented. Suppose now that $N$ is finitely presented. Then $N=q(L)$ for some finitely presented object $L$ and there are morphisms $\varphi: M \rightarrow L$ and $\psi: L \rightarrow M$ such that $\operatorname{id}_{M}-\psi \circ \varphi$ factors through some object in $\underline{\lim }_{\mathcal{C}}$. Thus $\operatorname{id}_{M}-\psi_{\circ} \varphi \in \operatorname{rad} \operatorname{End}(M)$ and therefore $\varphi$ is a split monomorphism. We conclude that $M$ is finitely presented.

The rest of this section is devoted to an application of the preceding result. Let us begin with an easy observation.

Lemma 4.2. Let $\mathcal{A}$ be a locally finitely presented category with products and sup- 
pose that $\mathcal{C}$ is a full additive subcategory of $\operatorname{fp}(\mathcal{A})$ which is covariantly finite. Then $\varliminf_{\mathrm{C}} \mathcal{C}$ is again locally finitely presented and has products. Moreover, if $\mathcal{A}$ satisfies any of the conditions (A1) - (A2), then $\lim _{\mathcal{C}}$ satisfies the same condition.

Proof. Clearly $\varliminf_{\mathrm{C}} \mathcal{C}$ is locally finitely presented. Furthermore it is closed under products taken in $\mathcal{A}$ by [8, Theorem 4.2] and has therefore products in itself. In fact $\underline{\lim \mathcal{C}}$ is a definable subcategory of $\mathcal{A}$ by [18, Proposition 4.13] and Zsp $\underline{\lim } \mathcal{C}$ can therefore be identified with a closed subset of Zsp $\mathcal{A}$. The second part of the assertion is now an immediate consequence.

We consider now the following example. Fix a triangular matrix ring

$$
\Lambda=\left[\begin{array}{cc}
A & { }_{A} M_{B} \\
0 & B
\end{array}\right]
$$

and we suppose for simplicity that $\Lambda$ is an artin algebra. As usual, we identify a $\Lambda$-module with a $B$-linear morphism $X \otimes_{A} M \rightarrow Y$ where $X \in \operatorname{Mod} A$ and $Y \in \operatorname{Mod} B$. Suppose now that $\mathcal{X}_{A} \subseteq \bmod A$ and $\mathcal{X}_{B} \subseteq \bmod B$ is a pair of full additive subcategories which are closed under direct summands. We denote by $\bmod (\Lambda, \mathcal{X})$ the full subcategory of finitely presented $\Lambda$-modules $X \otimes_{A} M \rightarrow Y$ with $X \in \mathcal{X}_{A}$ and $Y \in \mathcal{X}_{B}$. The full subcategory of $\Lambda$-modules $X \otimes_{A} M \rightarrow Y$ with $X \in \underline{\lim } \mathcal{X}_{A}$ and $Y \in \underline{\lim } \mathcal{X}_{B}$ is denoted by $\operatorname{Mod}(\Lambda, \mathcal{X})$. For example, $\operatorname{Mod}(\Lambda, \mathcal{X})$ is the category of prinjective $\Lambda$-modules in the sense of $[20,23]$ if $\mathcal{X}_{A}$ is the category of projective $A$-modules and $\mathcal{X}_{B}$ is the category of injective $B$-modules.

Lemma 4.3. Suppose that $\mathcal{X}_{A}$ and $\mathcal{X}_{B}$ are covariantly finite subcategories. Then $\bmod (\Lambda, \mathcal{X})$ is a covariantly finite subcategory of $\bmod \Lambda$ and the modules in $\operatorname{Mod}(\Lambda, \mathcal{X})$ are precisely the direct limits of modules in $\bmod (\Lambda, \mathcal{X})$. In particular $\operatorname{Mod}(\Lambda, \mathcal{X})$ is a locally finitely presented category with products satisfying (A1) - (A2) and $\operatorname{fp}(\operatorname{Mod}(\Lambda, \mathcal{X}))=\bmod (\Lambda, \mathcal{X})$.

Proof. To show that $\bmod (\Lambda, \mathcal{X})$ is a covariantly finite subcategory fix an arbitrary finitely presented $\Lambda$-module $X \otimes_{A} M \rightarrow Y$. We construct a left $\bmod (\Lambda, \mathcal{X})$ approximation as follows. Let $X \rightarrow X^{\prime}$ be the $\mathcal{X}_{A}$-approximation of $X$ and consider the following push-out in $\bmod B$.

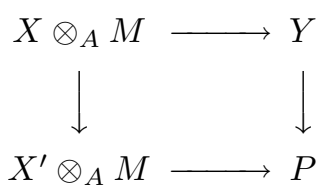

Composing $X^{\prime} \otimes_{A} M \rightarrow P$ with the $\mathcal{X}_{B}$-approximation $P \rightarrow Y^{\prime}$ then gives the approximation of $X \otimes_{A} M \rightarrow Y$. The full subcategory $\mathcal{A}$ of $\Lambda$-modules which are direct limits of modules in $\bmod (\Lambda, \mathcal{X})$ is locally finitely presented and has products 
by $[8$, Theorem 4.2$] \operatorname{since} \bmod (\Lambda, \mathcal{X})$ is covariantly finite. We leave it to the reader to verify that $\mathcal{A}=\operatorname{Mod}(\Lambda, \mathcal{X})$. (A1) - (A2) then follow from the preceding lemma since $\Lambda$ is an artin algebra. For example, $\{M\}$ is open for every finitely presented $M \in \operatorname{Zsp} \Lambda$ by [19, Lemma 4.4].

We give now our promised application of the preceding theorem. To this end the following definition is useful. A locally finitely presented category with products $\mathcal{A}$ is called generically wild if there is a generic object $M \in \mathcal{A}$ such that $\operatorname{End}(M) / \operatorname{rad} \operatorname{End}(M)$ is not a PI-ring. Note that in our example $\mathcal{A}=\operatorname{Mod}(\Lambda, \mathcal{X})$ the generic objects are precisely the generic $\Lambda$-modules contained in $\operatorname{Mod}(\Lambda, \mathcal{X})$.

Corollary 4.4. Let $\Lambda$ be a triangular matrix ring as above. Suppose that $\mathcal{X}_{A}$ and $\mathcal{X}_{B}$ are covariantly finite subcategories. Suppose also that $X \in \bmod (\Lambda, \mathcal{X})$ and let $\mathcal{C}=\operatorname{add} X$. Then the canonical functor $\operatorname{Mod}(\Lambda, \mathcal{X}) \rightarrow \varliminf_{\mathfrak{\operatorname { m o d }}} \underline{\operatorname{mob}}_{\mathcal{C}}(\Lambda, \mathcal{X})$ induces a bijection $M \mapsto M^{\prime}$ between the isomorphism classes of generic objects in $\operatorname{Mod}(\Lambda, \mathcal{X})$ and $\underline{\lim }_{\underline{\bmod }}(\Lambda, \mathcal{X})$ such that $\operatorname{End}(M) / \operatorname{rad} \operatorname{End}(M)$ and $\operatorname{End}\left(M^{\prime}\right) /$ $\operatorname{rad} \operatorname{End}\left(M^{\prime}\right)$ are isomorphic; in particular $\operatorname{Mod}(\Lambda, \mathcal{X})$ is generically wild if and only if $\varliminf_{\bmod }(\Lambda, \mathcal{X})$ is generically wild.

\section{The Auslander-Reiten translation}

Throughout this section we assume that the ring $\Lambda$ has a self-duality $D$ between $\bmod \Lambda$ and $\bmod \Lambda^{\mathrm{op}}$. We begin with some notation. Denote by $\operatorname{Proj} \Lambda$ the full subcategory of projective $\Lambda$-modules and let $\operatorname{proj} \Lambda=\operatorname{Proj} \Lambda \cap \bmod \Lambda$. Analogously, the categories $\operatorname{Inj} \Lambda$ and $\operatorname{inj} \Lambda$ are defined. Now define $\underline{\operatorname{Mod}} \Lambda=\operatorname{Mod} \Lambda\lceil\operatorname{Proj} \Lambda$ and $\underline{\bmod } \Lambda=\bmod \Lambda\lceil\operatorname{proj} \Lambda$; similarly let $\overline{\operatorname{Mod}} \Lambda=\operatorname{Mod} \Lambda\lceil\operatorname{Inj} \Lambda$ and $\overline{\bmod } \Lambda=$ $\bmod \Lambda\lceil\operatorname{inj} \Lambda$.

Our aim in this section is to discuss an equivalence $A: \underline{\operatorname{Mod}} \Lambda \rightarrow \overline{\operatorname{Mod}} \Lambda$ which we call the Auslander-Reiten translation. This functor coincides on the finitely presented level with the dual of transpose functor $D \operatorname{Tr}: \underline{\bmod } \Lambda \rightarrow \overline{\bmod } \Lambda$ which was introduced by Auslander and Reiten [4].

Some further definitions are needed. Denote by $\mathcal{P}$ the category of morphisms in $\operatorname{Proj} \Lambda$. More precisely, an object in $\mathcal{P}$ is a morphism $\pi: P \rightarrow Q$ between projective $\Lambda$-modules and the morphisms $\pi \rightarrow \pi^{\prime}$ in $\mathcal{P}$ for $\pi^{\prime}: P^{\prime} \rightarrow Q^{\prime}$ are given by pairs $(\alpha, \beta)$ of morphisms making the following diagram commutative.

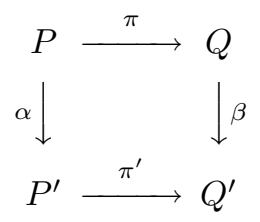

Clearly, the composition of $(\alpha, \beta): \pi \rightarrow \pi^{\prime}$ and $\left(\alpha^{\prime}, \beta^{\prime}\right): \pi^{\prime} \rightarrow \pi^{\prime \prime}$ is $\left(\alpha^{\prime} \circ \alpha, \beta^{\prime} \circ \beta\right)$. 
Lemma 5.1. The category $\mathcal{P}$ is a locally finitely presented category with products. An object $\pi: P \rightarrow Q$ in $\mathcal{P}$ is finitely presented iff $P$ and $Q$ are finitely presented.

Proof. The category $\operatorname{Proj} \Lambda$ is a locally finitely presented category with products. The assertion therefore follows from [1, Corollary 2.44].

We define now categories $\mathcal{P}^{\prime}$ and $\mathcal{P}^{\prime \prime}$ which have the same objects as in $\mathcal{P}$ but

$$
\operatorname{Hom}_{\mathcal{P}^{\prime}}\left(\pi, \pi^{\prime}\right)=\operatorname{Hom}_{\mathcal{P}}\left(\pi, \pi^{\prime}\right) /\left\{(\alpha, \beta) \mid \text { there is } \varphi: Q \rightarrow P^{\prime} \text { with } \pi^{\prime} \circ \varphi=\beta\right\},
$$

$\operatorname{Hom}_{\mathcal{P}^{\prime \prime}}\left(\pi, \pi^{\prime}\right)=$

$$
\operatorname{Hom}_{\mathcal{P}}\left(\pi, \pi^{\prime}\right) /\left\{(\alpha, \beta) \mid \text { there is } \varphi: Q \rightarrow P^{\prime} \text { with } \pi^{\prime} \circ \alpha=\pi^{\prime} \circ \varphi_{\circ} \pi=\beta \circ \pi\right\} .
$$

Lemma 5.2. The functor $\mathcal{P} \rightarrow \operatorname{Mod} \Lambda, \pi \mapsto$ Coker $\pi$, induces an equivalence $\mathcal{P}^{\prime} \rightarrow \operatorname{Mod} \Lambda$ and an equivalence $\mathcal{P}^{\prime \prime} \rightarrow \underline{\operatorname{Mod}} \Lambda$.

Proof. The cokernel functor is full and dense since every $\Lambda$-module admits a projective presentation. Moreover, given a morphism $(\alpha, \beta)$ in $\mathcal{P}$, we have $\operatorname{Coker}(\alpha, \beta)=$ 0 iff $(\alpha, \beta)$ lies in the kernel of the canonical functor $\mathcal{P} \rightarrow \mathcal{P}^{\prime}$, and $\operatorname{Coker}(\alpha, \beta)$ factors through a projective module iff $(\alpha, \beta)$ lies in the kernel of the canonical functor $\mathcal{P} \rightarrow \mathcal{P}^{\prime \prime}$.

We can now replace the projective $\Lambda$-modules in the definition of $\mathcal{P}, \mathcal{P}^{\prime}$ and $\mathcal{P}^{\prime \prime}$ with the injective $\Lambda$-modules and obtain in this way categories $\mathcal{I}, \mathcal{I}^{\prime}$ and $\mathcal{I}^{\prime \prime}$. Clearly we have the following analogue of the preceding lemma.

Lemma 5.3. The functor $\mathcal{I} \rightarrow \operatorname{Mod} \Lambda, \pi \mapsto \operatorname{Ker} \pi$, induces an equivalence $\mathcal{I}^{\prime} \rightarrow$ $\operatorname{Mod} \Lambda$ and an equivalence $\mathcal{I}^{\prime \prime} \rightarrow \overline{\operatorname{Mod}} \Lambda$.

Proof. Dualize the proof of the preceding lemma.

The following lemma is a reformulation of the fact that $\Lambda$ has a self-duality.

Lemma 5.4. The functor $-\otimes_{\Lambda} D \Lambda$ induces an equivalence $\operatorname{Proj} \Lambda \rightarrow \operatorname{Inj} \Lambda$.

Proof. An inverse is induced by the functor $\operatorname{Hom}_{\Lambda}(D \Lambda$,$) . The assertion is clear$ on the level of the finitely presented modules. The assertion follows for arbitrary $\Lambda$-modules since $\Lambda$ is an artinian ring having a self duality and therefore every projective (injective) module is a coproduct of finitely presented modules. Moreover, one uses the fact that $-\otimes_{\Lambda} D \Lambda$ commutes with coproducts. 
Suppose now that $M$ is an arbitrary $\Lambda$-module. We fix a minimal projective presentation $P \stackrel{\pi}{\rightarrow} Q \rightarrow M \rightarrow 0$ and a minimal injective copresentation $0 \rightarrow M \rightarrow$ $I \stackrel{\iota}{\rightarrow} J$. We call the kernel of $\pi \otimes_{\Lambda} D \Lambda$ the Auslander-Reiten translate of $M$ and

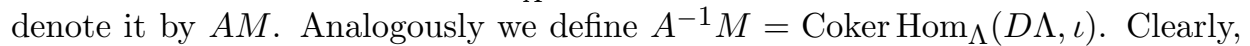
$A M$ and $A^{-1} M$ are unique only up to an isomorphism.

Proposition 5.5. The assignment $M \mapsto A M$ induces an equivalence $A$ : $\underline{\operatorname{Mod}} \Lambda \rightarrow$ $\overline{\operatorname{Mod}} \Lambda$. The restriction of $A$ to the finitely presented $\Lambda$-modules induces an equivalence $\underline{\bmod } \Lambda \rightarrow \overline{\bmod } \Lambda$.

Proof. The equivalence $\operatorname{Proj} \Lambda \rightarrow \operatorname{Inj} \Lambda$ induces an equivalence $\mathcal{P} \rightarrow \mathcal{I}$ and therefore also an equivalence $\mathcal{P}^{\prime \prime} \rightarrow \mathcal{I}^{\prime \prime}$. The assertion now follows from Lemma 5.2 and Lemma 5.3.

It follows from Proposition 2.1 that the canonical functor $\operatorname{Mod} \Lambda \rightarrow \underline{\lim \bmod } \Lambda$ induces a functor $p: \underline{\operatorname{Mod}} \Lambda \rightarrow \underline{\lim }_{\underline{\bmod }} \Lambda$. Analogously there is an induced functor $q: \overline{\operatorname{Mod}} \Lambda \rightarrow \underline{\lim } \overline{\bmod } \Lambda$.

Proposition 5.6. There is an equivalence $\underline{\lim \bmod } \Lambda \rightarrow \varliminf \overline{\bmod } \Lambda$ which makes the following diagram of functors commutative.

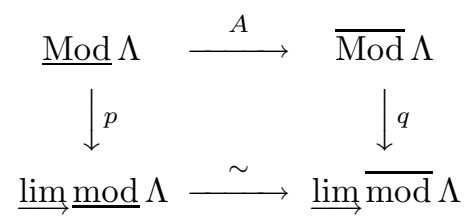

Proof. The equivalences $\mathcal{P} \rightarrow \mathcal{I}$ and $\underline{\operatorname{Mod}} \Lambda \rightarrow \overline{\operatorname{Mod}} \Lambda$ induce the following commutative diagram of functors

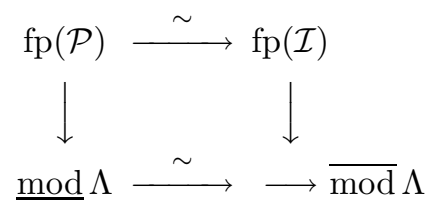

which extends by Lemma 1.1 to a commutative diagram of functors commuting with direct limits

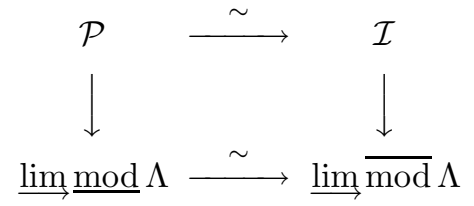


since $\mathcal{P}$ and $\mathcal{I}$ are locally finitely presented categories by Lemma 5.1. The assertion now follows from the fact that $A$ is induced by the equivalence $\mathcal{P} \rightarrow \mathcal{I}$.

We are interested in further properties of the Auslander-Reiten translation. To this end let us recall, without proofs, some well-known facts about projective presentations and injective copresentations.

Lemma 5.7. Let $P \stackrel{\pi}{\rightarrow} Q \rightarrow M \rightarrow 0$ be a projective presentation of a $\Lambda$-module M. Then there are decompositions $P=P_{0} \coprod P_{1} \amalg P_{2}$ and $Q=Q_{0} \coprod Q_{1} \coprod Q_{2}$ such that $\pi=\left[\begin{array}{ccc}\pi_{0} & 0 & 0 \\ 0 & \pi_{1} & 0 \\ 0 & 0 & \pi_{2}\end{array}\right]$ and

(i) $\pi_{0}$ induces a minimal projective presentation $P_{0} \stackrel{\pi_{0}}{\rightarrow} Q_{0} \rightarrow M \rightarrow 0$.

(ii) $\pi_{1}$ is an isomorphism.

(iii) $Q_{2}=0$.

Lemma 5.8. Let $0 \rightarrow M \rightarrow I \stackrel{\iota}{\rightarrow} J$ be an injective copresentation of a $\Lambda$-module M. Then there are decompositions $I=I_{0} \coprod I_{1} \coprod I_{2}$ and $J=J_{0} \coprod J_{1} \coprod J_{2}$ such that $\iota=\left[\begin{array}{ccc}\iota_{0} & 0 & 0 \\ 0 & \iota_{1} & 0 \\ 0 & 0 & \iota_{2}\end{array}\right]$ and

(i) $\iota_{0}$ induces a minimal injective copresentation $0 \rightarrow M \rightarrow I_{0} \stackrel{\iota_{0}}{\rightarrow} J_{0}$.

(ii) $\iota_{1}$ is an isomorphism.

(iii) $I_{2}=0$.

Lemma 5.9. Let $M$ be any $\Lambda$-module.

(1) A morphism $\pi: P \rightarrow M$ is a projective cover iff $P$ is projective and $\pi \otimes_{\Lambda} \Lambda / \operatorname{rad} \Lambda$ is an isomorphism.

(2) A morphism $\iota: M \rightarrow I$ is an injective envelope iff $I$ is injective and $\operatorname{Hom}_{\Lambda}(\Lambda / \operatorname{rad} \Lambda, \iota)$ is an isomorphism.

We are now in a position to collect the essential properties of the AuslanderReiten translate $A M$ of a module $M$. Of course, these properties are well-known for finitely presented modules [4].

Proposition 5.10. The assignment $M \mapsto A M$ induces a bijection between the isomorphism classes of $\Lambda$-modules without non-zero projective direct summand and the isomorphism classes of $\Lambda$-modules without non-zero injective direct summand. More precisely, the following holds for any $\Lambda$-module $M$ :

(1) AM has no non-zero injective direct summand.

(2) $A^{-1} M$ has no non-zero projective direct summand.

(3) $M=A^{-1} A M \coprod P$ for some projective $\Lambda$-module $P$.

(4) $M=A A^{-1} M \coprod I$ for some injective $\Lambda$-module $I$. 
Proof. The assertions (1) - (4) follow directly from Lemma 5.4, Lemma 5.7 and Lemma 5.8.

To study further properties of the Auslander-Reiten translate we need to recall the definition of a reduced product of a family of $\Lambda$-modules. Let $I$ be a non-empty set. Recall that a set $\mathcal{F}$ of non-empty subsets of $I$ is a filter on $I$ provided that for each pair of subsets $J_{1}, J_{2} \subseteq I$

(i) $J_{1}, J_{2} \in \mathcal{F}$ implies $J_{1} \cap J_{2} \in \mathcal{F}$;

(ii) $J_{1} \in \mathcal{F}$ and $J_{1} \subseteq J_{2}$ implies $J_{2} \in \mathcal{F}$.

Note, that the set $\mathcal{F}$ is directed if one defines $J_{1} \leq J_{2}$, if $J_{2} \subseteq J_{1}$.

Given a family $M_{i}, i \in I$, of $\Lambda$-modules and a filter $\mathcal{F}$ on $I$, the canonical morphisms $\prod_{i \in J_{1}} M_{i} \rightarrow \prod_{i \in J_{2}} M_{i}, J_{1} \leq J_{2}$ in $\mathcal{F}$, form a directed system and we denote by $\prod_{i \in I} M_{i} / \mathcal{F}=\varliminf_{J \in \mathcal{F}} \prod_{i \in J} M_{i}$ the reduced product of the $M_{i}$ 's with respect to $\mathcal{F}$.

Proposition 5.11. Let $M_{i}, i \in I$, be any family of $\Lambda$-modules and let $\mathcal{F}$ be a filter on $I$.

(1) $A \coprod_{i \in I} M_{i}=\coprod_{i \in I} A M_{i}$ and $A \prod_{i \in I} M_{i}=\prod_{i \in I} A M_{i}$.

(2) $A \prod_{i \in I} M_{i} / \mathcal{F}=\prod_{i \in I} A M_{i} / \mathcal{F}$.

Proof. The assertion is a consequence of the fact that taking direct limits and products commutes with taking minimal projective presentations. This follows from Lemma 5.9.

Corollary 5.12. The following holds for any $\Lambda$-module $M$.

(1) $M$ is pure-injective if and only if $A M$ is pure-injective.

(2) $M$ is $\Sigma$-pure-injective if and only if $A M$ is $\Sigma$-pure-injective.

(3) $M$ is endofinite if and only if $A M$ is endofinite.

Proof. (1) Recall that $M$ is pure-injective iff for every set $I$ the summation morphism $\coprod_{I} M \rightarrow M$ factors through the canonical morphism $\coprod_{I} M \rightarrow \prod_{I} M[14$, Proposition 7.32], see also [18, Theorem 2.6]. The proof of the preceding proposition then shows that $A M$ and $A^{-1} M$ are pure-injective if $M$ is pure-injective. If $A M$ is pure-injective, then $A^{-1} A M$ is pure-injective and therefore $M$ is pureinjective by Proposition 5.10 since every projective $\Lambda$-module is endofinite and therefore pure-injective.

(2) Recall that $M$ is $\Sigma$-pure-injective if any coproduct $\coprod_{I} M$ is pure-injective. The assertion now follows from part (1) and the fact that $A$ and $A^{-1}$ commute with coproducts.

(3) If $M$ is indecomposable, then $M$ is endofinite iff for every set $I$ there is a set $J$ such that $\prod_{I} M=\coprod_{J} M$. An arbitrary $\Lambda$-module $M$ is endofinite iff there are indecomposable endofinite modules $M_{1}, \ldots, M_{n}$ and sets $I_{1}, \ldots, I_{n}$ such that $M=\coprod_{i=1}^{n}\left(\coprod_{I_{i}} M_{i}\right)$ [7, Proposition 4.5]. The assertion now follows from the fact 
that $A$ and $A^{-1}$ commute with (co)products. In addition one uses the fact that $A$ and $A^{-1}$ preserve indecomposability which follows from Proposition 5.10 and Proposition 5.11.

Remark 5.13. The preceding results have analogues for $A^{-1}$.

Our next aim is to show that the Auslander-Reiten translation preserves the topology defined on Zsp $\Lambda$. We shall use the following characterization of a closed subset in Zsp $\Lambda$.

Lemma 5.14. The following conditions are equivalent for a subset $\mathcal{U}$ of $Z \operatorname{sp} \Lambda$.

(1) $\mathcal{U}$ is closed.

(2) Let $M=\prod_{i \in I} M_{i} / \mathcal{F}$ be any reduced product with $M_{i} \in \mathcal{U}$ for all $i \in I$. Then every indecomposable pure-injective direct summand of $M$ belongs to $\mathcal{U}$.

Proof. (1) $\Rightarrow(2)$. Let $\mathcal{X}$ be the definable subcategory of Mod $\Lambda$ corresponding to $\mathcal{U}$, i.e. $\mathcal{U}=\mathcal{X} \cap Z \operatorname{sp} \Lambda$. Then $\mathcal{X}$ is closed under taking reduced products and pure submodules and therefore (2) holds.

$(2) \Rightarrow(1)$. The full subcategory $\mathcal{X}$ of pure submodules of reduced products of modules in $\mathcal{U}$ forms the smallest definable subcategory containing $\mathcal{U}$ [18, Corollary 4.10]. It follows that $\mathcal{U}=\mathcal{X} \cap Z \operatorname{sp} \Lambda$ is closed.

Corollary 5.15. The assignment $M \mapsto A M$ induces a homeomorphism Zsp $\Lambda \backslash$ $\operatorname{Proj} \Lambda \rightarrow \operatorname{Zsp} \Lambda \backslash \operatorname{Inj} \Lambda$.

Proof. It follows from Proposition 5.10, Proposition 5.11 and Corollary 5.12 that $M \mapsto A M$ induces a bijection Zsp $\Lambda \backslash \operatorname{Proj} \Lambda \rightarrow \operatorname{Zsp} \Lambda \backslash \operatorname{Inj} \Lambda$. Using the preceding lemma together with the fact that $A$ preserves reduced products, one shows that $A$ preserves also the topology defined on $\operatorname{Zsp} \Lambda$.

Corollary 5.16. Let $\Lambda$ be a finite dimensional algebra over an algebraically closed field. If $\Lambda$ has tame representation type, then $A M=M$ for every generic $\Lambda$-module $M$.

Proof. It has been shown in [19, Corollary 9.8] that for every generic $\Lambda$-module $M$ there is an $A$-invariant subset $\mathcal{T}$ of finite dimensional modules in $Z \operatorname{sp} \Lambda$ such that $M$ is the unique generic module in the Ziegler closure $\overline{\mathcal{T}}$. Clearly, $\overline{\mathcal{T}}$ is also $A$-invariant by the preceding result and therefore $A M=M$ since $A$ preserves endofiniteness by Corollary 5.12. 


\section{Triangulated categories with direct limits}

Examples of rings having a self-duality are quasi-Frobenius rings, i.e. those rings where projective and injective $\Lambda$-modules coincide. The stable categories $\underline{\bmod } \Lambda$ and $\underline{\operatorname{Mod}} \Lambda$ of a quasi-Frobenius ring carry an additional structure; they are triangulated in the sense of Verdier $[22,15]$. Given a triangulated category $\mathcal{C}$ one can form the category Ind $\mathcal{C}$ of ind-objects in the sense of Grothendieck and Verdier [12]. However, it is not clear under which circumstances the triangulation in $\mathcal{C}$ induces a triangulation in Ind $\mathcal{C}$. The following result shows that in some non-trivial cases the answer to this question is positive.

Theorem 6.1. Let $\Lambda$ be a quasi-Frobenius ring of finite representation type. Then $\underline{\operatorname{Mod}} \Lambda$ is a triangulated category in which direct limits exist. Moreover, the inclusion $\underline{\bmod } \Lambda \rightarrow \underline{\operatorname{Mod}} \Lambda$ induces an equivalence between $\operatorname{Ind} \underline{\bmod } \Lambda$ and $\underline{\operatorname{Mod}} \Lambda$.

Proof. The first part of the assertion follows from Corollary 2.3. In fact, there it is shown that $\underline{\lim } \underline{\bmod } \Lambda$ and $\underline{\operatorname{Mod}} \Lambda$ are equivalent. The second part of the assertion follows from our definition of $\underline{\lim } \underline{\bmod } \Lambda$, since Flat $\left.(\underline{\bmod } \Lambda)^{\mathrm{op}}, \mathrm{Ab}\right)$ and Ind $\underline{\bmod } \Lambda$ are canonically equivalent [8].

\section{References}

[1] J. Adámek and J. Rosický, Locally presentable categories and accessible categories, London Math. Soc. Lec. Note Series 189 (1994).

[2] I. Assem and J.A. de la Peña, On the tameness of trivial extension algebras, Fund. Math. 149 (1996), 171-181.

[3] M. Auslander, Representation theory of artin algebras II, Comm. in Algebra 1 (1974), 269-310.

[4] M. Auslander and I. Reiten, Representation theory of artin algebras III, Comm. Algebra 3 (1975), 239-294.

[5] W. Crawley-Boevey, On tame algebras and bocses, Proc. London Math. Soc. 56 (1988), 451-483.

[6] W. Crawley-Boevey, Tame algebras and generic modules, Proc. London Math. Soc. 63 (1991), 241-264.

[7] W. Crawley-Boevey, Modules of finite length over their endomorphism ring, in: Representations of algebras and related topics, eds. S. Brenner and H. Tachikawa, London Math. Soc. Lec. Note Series 168 (1992), 127-184.

[8] W. Crawley-Boevey, Locally finitely presented additive categories, Comm. Algebra 22 (1994), 1644-1674.

[9] Yu.A. Drozd, Tame and wild matrix problems. Representations and quadratic forms, Institute of Mathematics, Academy of Sciences Ukrainian SSR, Kiev (1979), 39-74, Amer. Math. Soc. Transl. 128 (1986), 31-55.

[10] K. Erdmann, On Auslander-Reiten components for group algebras, J. Pure Appl. Algebra 104 (1995), 149-160.

[11] P. Gabriel, Des catégories abéliennes, Bull. Soc. math. France 90 (1962), 323-448.

[12] A. Grothendieck and J.L. Verdier, Prefaisceaux, in: Théorie des Topos et Cohomologie 
Etale des Schémas, Springer Lec. Notes 269 (1972), 1-217.

[13] L. Gruson and C.U. Jensen, Deux applications de la notion de L-dimension, C. R. Acad. Sci. Paris Ser. A 282 (1976), 23-24.

[14] C.U. Jensen and H. Lenzing, Model Theoretic Algebra, Gordon and Breach, New York 1989.

[15] D. Happel, On the derived category of a finite dimensional algebra, Comment. Math. Helv. 62 (1987) 339-389.

[16] H. Krause, The spectrum of a locally coherent category, J. Pure Appl. Algebra 114 (1997) 259-271.

[17] H. Krause, Functors on locally finitely presented categories, Colloq. Math., to appear.

[18] H. Krause, Exactly definable categories, J. Algebra, to appear.

[19] H. Krause, Generic modules over artin algebras, Proc. London Math. Soc., to appear.

[20] J.A. de la Peña and D. Simson, Prinjective modules, reflections functors, quadratic forms, and Auslander-Reiten sequences, Trans. Amer. Math. Soc. 329 (1992), 733-753.

[21] M. Schmidmeier, Auslander-Reiten Köcher für artinsche Ringe mit Polynomidentität, Diss. Univ. München (1996).

[22] J.L. Verdier, Catégories derivées, état 0, Springer Lec. Notes 569 (1977), 262-311.

[23] D. Vossieck, Représentations de bifuncteurs et interprétation en termes de modules, $C . R$. Acad. Sci. Paris Ser. A 307 (1988), 713-716.

[24] M. Ziegler, Model theory of modules, Ann. of Pure and Appl. Logic 26 (1984), 149-213.

Henning Krause

Fakultät für Mathematik

Universität Bielefeld

D-33501 Bielefeld, Germany

e-mail: henning@mathematik.uni-bielefeld.de

(Received: July 24, 1996) 